\title{
OBSERVATORIO
}

\section{UNIR EDUCACIÓN Y BIBLIOTECAS: LA EVOLUCIÓN DE UN RETO PERMANENTE}

José-Antonio Gómez-Hernández

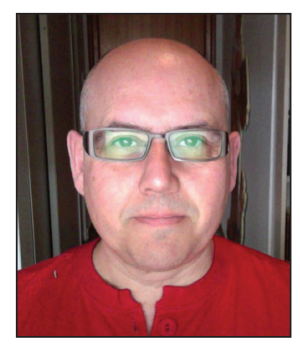

José-Antonio Gómez-Hernández es catedrático de biblioteconomía en la Universidad de Murcia. Doctor en filosofía, comenzó como bibliotecario en 1984 en esta Universidad, de la que ha sido decano de la Facultad de Documentación, director de su editorial, Editum, y del Servicio de Cultura. Ha publicado sobre los servicios educativos y de alfabetización informacional de las bibliotecas, así como sobre gestión y promoción de las mismas.

http://orcid.org/0000-0003-4532-1142

Univ. de Murcia, Fac. de Comunicación y Documentación Campus de Espinardo, 30071 Murcia, España jgomez@um.es

\section{Resumen}

Valoración de los servicios educativos de las bibliotecas tanto escolares como públicas y universitarias en España. Se identifican posibilidades y retos para intensificar la implicación y el apoyo bibliotecario al aprendizaje en los distintos niveles educativos. Destacan aspectos como la necesidad de cambio en la cultura docente y la integración bibliotecaria en los espacios virtuales del e-learning.

\section{Palabras clave}

Bibliotecas escolares, Bibliotecas universitarias, Aprendizaje permanente, E-learning.

\section{Title: Merging education and libraries: the evolution of a permanent challenge}

\section{Abstract}

An assessment is made of educational services provided by school, public and university libraries in Spain. Opportunities and challenges to increase the involvement and support of librarians at the various educational levels are identified. Aspects such as the need for change in the teaching culture and the integration of libraries in e-learning are highlighted.

\section{Keywords}

School libraries, University libraries, Lifelong learning, E-learning.

Gómez-Hernández, José-Antonio (2013). “Unir educación y bibliotecas: la evolución de un reto permanente”. El profesional de la información, marzo-abril, v. 22, n. 2, pp. 101-105.

\section{Introducción}

El profesional de la información dedica su tema central a las relaciones y formas de conexión de las bibliotecas con la educación. El fin es homenajear a la revista que nació para luchar contra la "incomprensible marginación educativa de la biblioteca" -en palabras de su fundador Francisco J. Bernal. Educación y biblioteca acompañó durante veinte años los esfuerzos de los bibliotecarios para ofrecer oportunidades de aprendizaje. Por ello, recordarla es de justicia, y además relevante, porque los servicios educativos de las instituciones documentales tienen una enorme vigencia y un mayor futuro, a pesar de la complejidad del sistema educativo y sus muchas dificultades y contradicciones. No es sólo una utopía de renovación pedagógica, sino además una oportunidad profesional a nuestro alcance.
Usar una biblioteca es siempre una experiencia de aprendizaje, en la que se pueden adquirir hábitos y valores (de lectura, de compartir información, de respetar a los demás, de participar, desear estar al día o ser crítico con la información), conceptos (los relativos a la sociedad de la información, la cultura heredada y la digital), y procedimientos (la búsqueda, valoración y comunicación de información, la interrelación de informaciones y documentos o la resolución de problemas de comprensión de los textos). Desde que accede a una biblioteca, el lector está aprendiendo: al leer amplía sus conocimientos, su cultura, o su capacidad de comprensión; al estudiar en la biblioteca está aprovechándola para sus actividades de educación formal; al informarse está obteniendo los elementos para producir conocimiento; al acceder a documentos de la biblioteca está logrando fuentes de conocimiento seleccionadas y contrastadas; al 


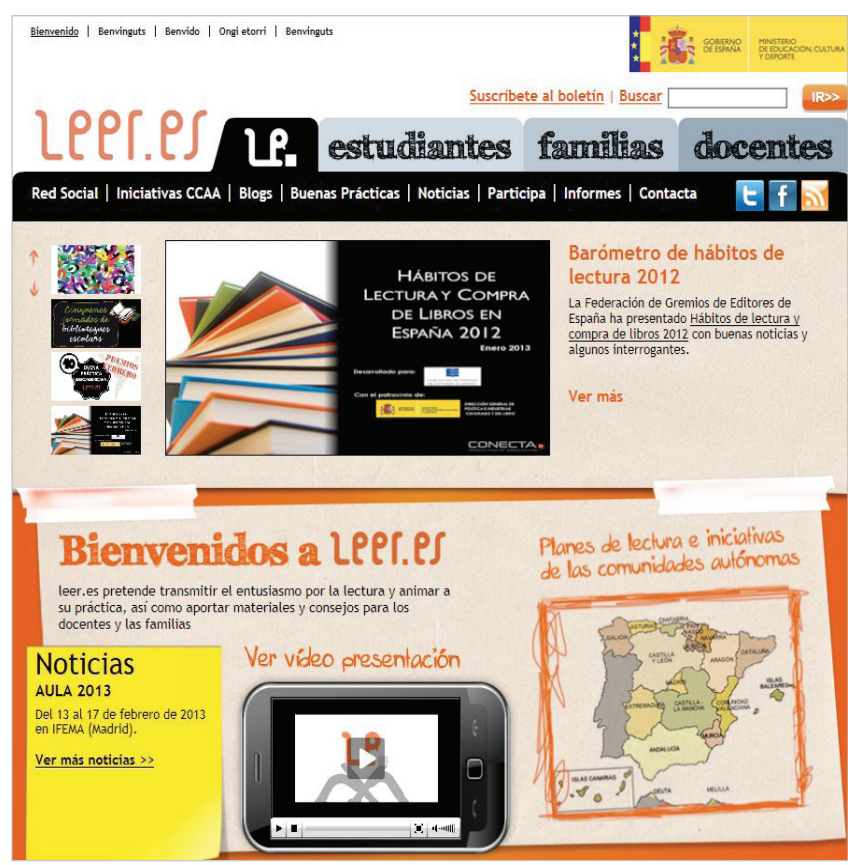

http://leer.es

divertirse, acceder a la cultura o usar tecnología para la comunicación o el ocio está adquiriendo alfabetización digital o conociendo distintas manifestaciones artísticas; en suma, al usar la biblioteca junto a otros está adquiriendo hábitos democráticos y de respeto, interiorizando las prácticas de cooperación, formando parte de comunidades de aprendizaje y ampliando su visión del mundo de la información (Gómez-Hernández, 2004). Y hay un paralelismo entre el aprendizaje como procesamiento de información y la labor bibliotecaria, que la hace referente y modelo para los usuarios: "las bibliotecas buscan, evalúan, seleccionan, clasifican y organizan la información para su difusión, y esto es lo que hacemos cuando aprendemos: buscar información a partir de una necesidad, localizarla, evaluarla, seleccionar o extraer la relevante, organizarla en nuestro sistema de conocimientos previos, y aplicarla a nuestros fines o comunicarla".

La relación educación-biblioteca se materializa de modo diferente en tres contextos, cuya situación valoraremos: el de la educación básica y bachillerato, el de la educación superior, y el del aprendizaje permanente en el marco de la biblioteca pública.

\section{Obstáculos estructurales y posibilidades de las bibliotecas en la educación básica}

En colegios e institutos lograr una simbiosis efectiva de bibliotecas y educación es una tarea titánica y propia de Sísifo, por los retrocesos que suelen seguir a los avances logrados con tesón. Entre lo positivo puede recordarse:

- Reconocimiento del carácter básico y transversal de la competencia informacional y digital en la educación secundaria, para cuya adquisición se han propuesto modelos y proyectos (Durban, 2011; Blasco; Durban-Roca, 2012; Durban-Roca; Cid; García-Guerrero, 2012; Piquín, 2012...).

- Fomento de buenas prácticas y experiencias en bibliotecas escolares a través de programas de impulso (Durban, 2011; Miret et al., 2010), premios, servicios coordinado- res de apoyo como los de Andalucía o Catalunya, y guías organizativas y de evaluación (Miret et al., 2011). Hacer visibles los éxitos, mostrar que son posibles y ayudar a extenderlos son tareas fundamentales para conseguir el protagonismo educativo de las bibliotecas.

Difusión de trabajos, tesis doctorales, propuestas de aplicación (webquests -sistema web de aprendizaje e investigación-, edublogs o proyectos documentales...) y portales con recursos didácticos, software educativo y actividades de promoción lectora. Los hay tanto autonómicos como estatales -Leer.es, Sol-e.com- y de fundaciones -Educared de Fundación Telefónica, redes de la Organización de Estados Iberoamericanos... Y junto a ellos muchas comunidades virtuales en el ámbito de las tecnologías del aprendizaje y la cooperación, que se han interesado por la renovación pedagógica, la integración en el currículo de las competencias informacionales y los contenidos multimedia.

\section{http://leer.es}

http://sol-e.com

http://www.educared.org

http://www.oei.es

- Mejora de la formación de los profesores en tecnologías educativas y competencias informacionales, así como la de muchos bibliotecarios escolares. Aunque no sean graduados en información y documentación, a los que se sigue ignorando en este campo profesional, los maestros que ejercen en las bibliotecas han obtenido una capacitación básica, se les ha facilitado sistemas integrados de gestión, cuentan con manuales de organización y con guías de utilización de la biblioteca escolar. Todo esto debemos considerar que contribuye a desarrollar un aprendizaje más activo y basado en fuentes, más allá del predominante libro de texto -impreso o digital.

\section{Los servicios educativos de las institucio- nes documentales tienen una enorme vigencia y un mayor futuro}

Pero hay muchos problemas, consecuencia de la gran complejidad de la educación, y que explican que la biblioteca no cobre protagonismo suficiente. La educación es muchas cosas a la vez: un derecho fundamental y una necesidad de las personas a lo largo de toda su vida; una cuestión desafortunadamente ideologizada, y una actividad económica susceptible de convertirse en negocio o fuente de beneficios... Es un proceso mediado por múltiples factores, como el mercado laboral, los medios económicos disponibles, la legislación educativa y la ideología predominante, la formación del profesorado, las actitudes familiares y sus circunstancias, y por supuesto, las expectativas y formas de aprender de los estudiantes. La educación -y su materialización en sistemas educativos y organizaciones de enseñanza- se ha cuestionado siempre, pero en nuestra época si cabe más, por muchas razones:

- Los métodos pedagógicos se muestran arcaicos frente la sociedad de la información y las tecnologías de la comunicación. E igualmente se cuestionan los contenidos porque 
las expectativas de aprendizaje han cambiado, y los estudiantes no ven la relevancia de algunos de los saberes que se imparten;

\section{$\mathrm{SOL}$ de Lectura}

- modelos neoliberales que aspiran a trasladar buena parte de los procesos educativos al sector privado, y la crisis económica está haciendo peligrar el acceso a la educación de calidad a los sectores desfavorecidos y excluidos;

- hay una tensión mayor que nunca entre el mercado laboral y el sistema educativo, condicionando cada vez más lo que se enseña en las aulas. Sobre todo falta la capacidad para llegar a pactos políticos perdurables para su organización, pues los gobiernos modifican en cuanto pueden las leyes educativas, por intereses sectarios ${ }^{1}$.

En esta situación convulsa falta estabilidad y

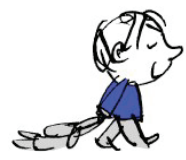

Marzo de 2013
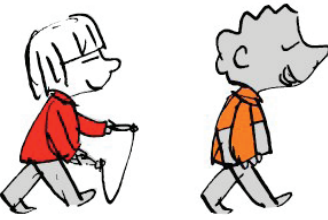

Familias

Biblioteca familiar | ¡Cuántas cosas para hacer! | Asesoría | Ideas | Lecturas SOL

\section{Banco de Recursos para Profesionales}

Agenda | Directorio | Actividades | Publicaciones |

Especialistas@| Museo SOL | Lecturas SOL

http://sol-e.com condiciones para que la biblioteca se integre en los centros escolares. Tanto las familias como los políticos de la educación, los alumnos y los profesores ven otros problemas antes que el de hacer de la biblioteca una mediación básica de la escuela. Y con la reducción de la financiación ello se ha hecho más evidente, al cuestionarse servicios elementales como el comedor escolar, el transporte o los profesores de apoyo a los alumnos con necesidades educativas especiales. Hay cansancio y estrés en el profesorado, que necesita actualizar su formación y tener apoyos que no recibe; hay problemas de aprendizaje en el alumnado que requiere un apoyo imposible de dar si no hay medios; escasea la dotación de colecciones y tecnologías para que las bibliotecas en los centros escolares tengan posibilidad de usos relevantes en la enseñanza; la figura del bibliotecario no está reconocida bien en la legislación escolar, y los proyectos piloto están en precario si no reciben la ayuda que haría factible su consolidación, interrumpiéndose cuando los bibliotecarios escolares se trasladan o dejar de tener las suficientes horas de dedicación.

La relación educación-biblioteca se materializa en tres contextos: educación básica y bachillerato, educación superior, y aprendizaje permanente

El riesgo es que, entre controversias, recortes y prioridades que se anteponen, la biblioteca escolar se pueda ir viendo superflua o reemplazable. Cuando, a la vez, las tecnologías, las redes de información y contenidos multimedia aportan también al sistema educativo información, interactividad, facilidad de acceso y posibilidades didácticas. El pragmatismo de los docentes les lleva a buscar soluciones que mejoren su docencia pero que no impliquen tantos esfuerzos como los que exige el mantenimiento de bibliotecas escolares dinámicas. Por tanto, o a éstas se las respalda y funcionan para hacer más sencilla y eficiente la labor al profesor, o seguirán marginadas.

Creemos que las bibliotecas son un elemento clave para la función transformadora que la educación debe tener, una vía para resolver muchas de las carencias que conducen al fracaso escolar y a los pobres resultados escolares, al posibilitar la interacción y el aprendizaje dialógico². Es una utopía que tiene que ver con lo que querríamos conseguir, pero soñar es también pragmático, pues sin sueños no se puede mejorar la realidad, sino que meramente se la reproduce.

\section{Bibliotecas en la educación superior}

Como la escuela, la universidad ha vivido los últimos años en permanente agitación: la adaptación al Espacio europeo de educación superior ha reducido la duración de los grados iniciales, ha favorecido el aumento de los posgrados con un encarecimiento considerable de las matrículas-, ha estimulado cierta renovación de los métodos docentes, y generalizado los campus virtuales a través de los cuales se imparten cada vez más programas e-learning.

Las bibliotecas universitarias ya tenían desde fines de los años ochenta estructuras muy fuertes que las han ayudado a adaptarse a los cambios de la educación superior, intentando vincularse cada vez más a los objetivos de sus instituciones. Ello las ha llevado a potenciar sus servicios educativos, intentando protagonizar la enseñanza de competencias informacionales -en alianza con los servicios informáticos-, colaborando con los profesores en su impartición, o programando autónomamente cursos contextualizados según las demandas de estudiantes de distintos niveles.

Rebiun ha extendido el modelo de biblioteca como crai y los programas de alfabetización informacional. Se han sistematizado modelos y manuales de formación, se ha incluido en los planes la actualización de profesores y bibliotecarios, y se intenta participar en la docencia. En menos de treinta años han desaparecido posibles reticencias de los bibliotecarios a ser mediadores en el aprendizaje universitario, y se han entusiasmado con la tarea de enseñar o de contribuir a que los estudiantes aprendan mejor con su apoyo.

Un elemento clave para la evolución de las bibliotecas universitarias es cómo se ha multiplicado -igual que en la educación básica- la oferta de contenidos. Hay millones de objetos de aprendizaje disponibles para los universitarios en las redes, tanto atomizadas como estructurados en forma de cursos. Se publican por el interés en apoyar el conocimien- 
to abierto de muchas universidades, y por su función de marketing de los programas formativos. Por una parte, los repositorios digitales abiertos mantenidos por las bibliotecas son una fuente enorme de contenidos educativos. Pero, pero más allá de ellos, el fenómeno de los open course ware (ocw, materiales de enseñanza gratuitos de nivel universitario) y más recientemente los moocs (massive open online courses), cursos online abiertos que pueden ser seguidos masivamente, ha hecho casi inabarcable la oferta accesible de educación superior. Las universidades compiten por atraer la atención de los estudiantes tanto hacia su oferta básica de grado y posgrado como a la formación e-learning.

Por ello, si las bibliotecas quieren seguir contribuyendo a los fines de sus universidades, deben integrarse al máximo en estas modalidades formativas, en las que tendrían que actuar como consultoras, educadoras y proveedoras de información de calidad. Por ahora echamos de menos una presencia más activa y relevante en los campus virtuales -incluso directamente en las webs de las asignaturas para ejercer roles de asesor y referencia digital-, dado que son el escenario de buena parte de las actividades de aprendizaje de los grados y posgrados. No es sencillo: a veces los profesores tienden a facilitar todos los materiales docentes en el campus virtual, y con ello pueden estar haciendo poco necesario para el estudiante medio el buscar más información, empobreciendo el uso de las bibliotecas y la adquisición de competencias informacionales. Por eso hay que ayudar mucho al profesorado en el diseño de contenidos y actividades que impliquen los recursos digitales que las bibliotecas pueden ofrecer y canalizar. Y eso conlleva el reto de interactuar más con otros agentes como las unidades de innovación educativa, las editoriales universitarias o los servicios de difusión de los resultados de investigación.

Y la otra dificultad que creemos que subsiste para que tenga sentido esta labor educativa es que todavía no se dominan los métodos para evaluar las capacidades informacionales

You Tube

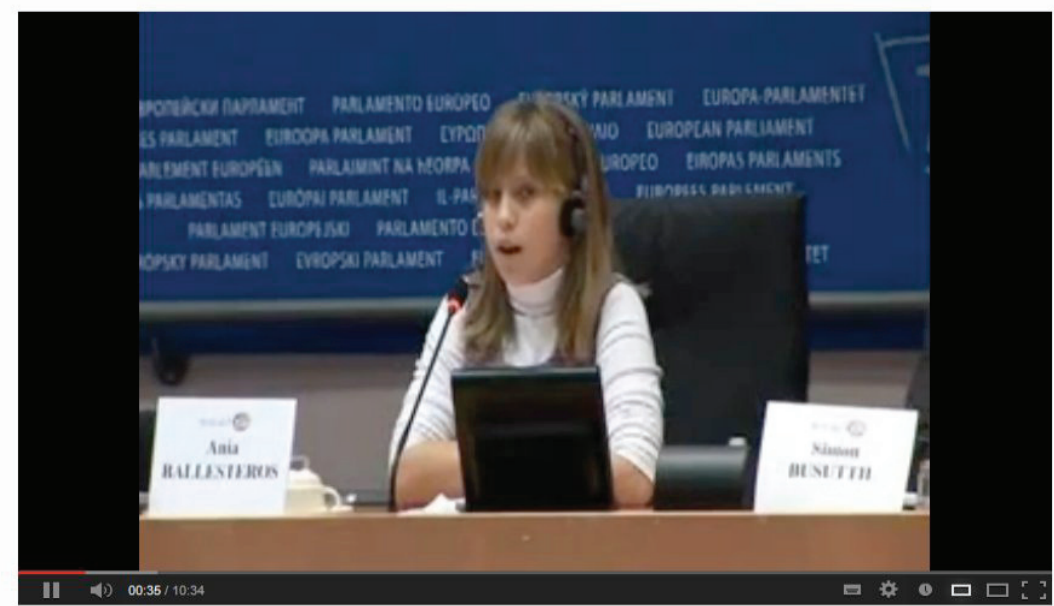

Ania Ballesteros [Comunitat d'aprenentage Montserrat de Terrassa]

Parlamento Europeo, Bruselas. Conferencia final del proyecto Includ-Ed "Actuaciones de éxito para superar la exclusión educativa en Europa" en la sede del en Bruselas. Una alumna de 10 años cuenta el funcionamiento de los grupos interactivos y las tertulias literarias dialógicas de su escuela de Terrassa (Barcelona). Diciembre 2011.

http://youtu.be/F-2zOJ6-ni8 alcanzadas por los estudiantes, con un modelo coherente con el enfoque competencial. Evaluar condiciona y orienta la labor de aprendizaje; aunque no sea un fin en sí mismo, necesitamos que se puedan acreditar las competencias logradas para que se valore la formación realizada por los bibliotecarios.

\section{Estamos en una situación convulsa en la que falta estabilidad y condiciones para que la biblioteca se integre en los cen- tros escolares}

Diríamos en síntesis que las bibliotecas han sabido adaptarse a la evolución de la educación superior, pero que deben seguir haciéndolo para integrarse al máximo en la enseñanza universitaria presencial y virtual. Integrar más los materiales de los repositorios digitales y los ocw entre los recursos de aprendizaje, asesorar a estudiantes y a profesores en las actividades programadas para que los trabajos estudiantiles impliquen las destrezas informaciones, ser líderes y estar atentos para participar en la innovación pedagógica... Se trata de seguir soñando en el nivel de la educación superior, más allá de los intentos de mercantilización que sufrimos actualmente.

\section{Biblioteca pública y aprendizaje informal y permanente}

La biblioteca pública asume su reto educativo, colaborando con la escuela o con el aprendizaje autodidacta, favoreciendo el acceso a la lectura y sus hábitos desde la infancia a la vejez, promoviendo el conocimiento de la literatura y las manifestaciones culturales, siendo ese espacio ciudadano abierto para todos. Los clubes de lectura o sus programas de alfabetización informacional y de otras competencias básicas son muestras de su función educativa. Alegra mucho ver que la biblioteca pública colabora con la escolar, se dan clases de repaso, o que se imparten en ella talleres de una gran diversidad de temas: desde los huertos ecológicos al retoque fotográfico, la búsqueda de empleo, la realidad aumentada o la gestión del tiempo. Y emociona ver en una cola para llevarse libros de una biblioteca pública cómo coinciden niños, jóvenes o ancianos, personas que pueden ser mendigos, intelectuales o camareros, todos con el deseo de leer, escuchar o acceder a cualquier registro de la cultura humana. Y que han encontrado en la biblioteca la oportunidad de hacerlo: un ambiente familiar y acogedor, un estímulo al saber. Una biblioteca llena de usuarios buscando individualmente o con otros algo de la cultura humana para saber más, o para aprender de lo que otros construyeron es un reflejo de una sociedad mejor que quisiéramos tener. 
La cuestión aquí es lograr más visibilidad: ante la demanda de aprendizaje permanente, muchísimas entidades ofrecen formación, y se trata de colaborar para compartir recursos escasos y conectar juntos con los públicos receptores comunes, afirmando nuestro espacio.

\section{Reflexiones finales}

Un reto común es cómo interiorizar las bibliotecas en la cultura docente, una compleja amalgama de hábitos, prácticas y normas más o menos conscientes. A ello se oponen la división rígida de horarios y currículo, la compactación de las jornadas escolares, la visión instrumental de las competencias, la anteposición de todo tipo de intereses, el uso exclusivo del hilo conductor del libro de texto y apuntes cerrados, o las formas de evaluación. Son obstáculos que impiden hacer realidad el ideal bibliotecario.

\section{Las bibliotecas deben integrarse al máxi- mo en las modalidades formativas como consultoras, educadoras y proveedoras de información de calidad}

Los cambios se producen, se asimilan y se adoptan muy lentamente. Para conseguirlos debemos insistir en la formación inicial y permanente de docentes y bibliotecarios. Debemos difundir al máximo los buenos ejemplos de bibliotecas que se aproximen al ideal pretendido, para que funcionen como centros de difusión de la innovación y ayuden a visualizar su posibilidad y beneficios (Gómez-Hernández, 2006). Debemos perseguir proyectos integrados y coherentes en los centros docentes, no exclusivos o que afecten sólo a las bibliotecas, donde se recoja su carácter axial para el desarrollo de los proyectos curriculares y las programaciones didácticas.

Hay obstáculos para el ensamblaje soñado de bibliotecas y educación, pero también muchas materializaciones y avances. Se debe defender el optimismo de la voluntad frente a las razones para el pesimismo, utilizando la expresión de Gramsci. La inteligencia, la creatividad, la curiosidad y la innovación deben ayudarnos a superar problemas, encontrar tendencias, nichos de interés y soluciones viables, sostenibles y útiles para contribuir a la educación, dentro o fuera de nuestras bibliotecas. Sigamos trabajando en el presente por esa integración de educación y bibliotecas, para construir la realidad futura a que aspiramos.

\section{Notas}

1. Como está pasando ahora: se quiere aprobar una nueva ley, la Lomce (Ley orgánica para la mejora de la calidad educativa), sin consenso posible, pues sus prioridades son "recentrar" los contenidos para potenciar el nacionalismo español sobre los nacionalismos periféricos, el apoyo a la enseñanza privada y elitista, la diferenciación del alumnado en itinerarios académicos distintos a partir de los 12 ó 13 años, y restablecer la religión católica como asignatura.

2. Uno de estos ejemplos es el de las comunidades de aprendizaje que se han creado en centros escolares en contextos de marginación y gran diversidad: a partir del trabajo en bibliotecas tutorizadas y aulas digitales, con la ayuda de miembros de la comunidad y las familias, los estudiantes aprendieron a través de tertulias literarias dialógicas y otras actividades, que mejoraron muchísimo su rendimiento escolar. Con la lectura en común y el diálogo sobre los clásicos de la literatura universal aumentó su competencia lectora y se contribuyó a transformar el contexto social e incluso sus vidas (Díaz-Palomar; Flecha, 2010) (fig. 2).

\section{Referencias}

Blasco-Olivares, Anna; Durban-Roca, Glòria (2012). "La competencia informacional en la enseñanza obligatoria a partir de la articulación de un modelo específico". Revista española de documentación científica, pp. 100-135.

http://redc.revistas.csic.es/index.php/redc/article/ view/746

http://dx.doi.org/10.3989/redc.2012.mono.979

Díez-Palomar, Javier; Flecha-García, Ramón (2010) “Comunidades de aprendizaje: un proyecto de transformación social y educativa". Revista interuniversitaria de formación del profesorado, n. 67, pp. 19-30.

http://www.aufop.com/aufop/uploaded_files/ revistas $/ 1268689288 . p d f$

Durban-Roca, Glòria (2011). El papel de la biblioteca escolar hoy. Panorama actual de las bibliotecas escolares en España. Buenas prácticas y centros premiados. Presentación ppt. http://www.bibliotecaescolar.info/buenaspracticas.pdf

Durban, Glòria; Cid, Ana; García Guerrero, José (2012) Programas para el desarrollo de la competencia informacional articulados desde la biblioteca escolar. Sevilla: Junta de Andalucía.

http://www.juntadeandalucia.es/educacion/webportal/ abaco-portlet/content/9e344cda-a851-46bb-b6e107aced30d800

Gómez-Hernández, José-Antonio (2004). “Evolución conceptual de la función educativa de la biblioteca pública". Boletín de Anabad, v. 54, n. 1-2, p. 807-814. http://www.anabad.org/archivo/docdow.php?id=251

Gómez-Hernández, José-Antonio (2006). “Cultura escolar y biblioteca". Cuadernos de pedagogía, n. 353, p. 93.

Miret, Inés; Baró, Mònica; Mañá, Teresa; Vellosillo, Inmaculada; Montero, Ignacio (2010). Bibliotecas escolares "entre comillas". Estudio de casos: buenas prácticas en la integración de la biblioteca en los centros educativos. Madrid: Secretaría General Técnica del Ministerio de Educación, y Fundación Germán Sánchez Ruipérez. http://docentes.leer.es/files/2010/09/entre_comillas.pdf

Miret, Inés; Baró, Mònica; Mañá, Teresa; Vellosillo, Inmaculada (2011). Bibliotecas escolares ¿entre interrogantes? Herramienta de autoevaluación. Preguntas e indicadores para mejorar la biblioteca. Madrid: Ministerio de Educación, Fundación Germán Sánchez Ruipérez.

http://www.lecturalab.org/uploads/website/docs/2684-2Bibliotecas_escolares_entre_interrogantes.pdf

Piquín, Rosa (2012). Proyectos documentales integrados. Navarra: Gobierno de Navarra, Departamento de Educación. http://dpto.educacion.navarra.es/publicaciones/pdf/ PDIcastell.pdf 